University of Nebraska - Lincoln

DigitalCommons@University of Nebraska - Lincoln

\title{
$7-2012$
}

\section{Fourier transform infrared spectroscopy and wide-angle X-ray scattering: Investigations on polypropylene-vapor-grown carbon nanofiber composites}

\author{
Mircea Chipara \\ University of Texas Pan American, mchipara@utpa.edu \\ John Hamilton \\ University of Texas Pan American \\ Alin Cristian Chipara \\ University of Texas Pan American, cristian.chipara@gmail.com \\ Tom George \\ University of Nebraska-Lincoln \\ Dorina Magdalena Chipara \\ University of Texas Pan American, dorina.chipara@utrgv.edu \\ See next page for additional authors \\ Follow this and additional works at: https://digitalcommons.unl.edu/physicssellmyer \\ Part of the Physics Commons
}

Chipara, Mircea; Hamilton, John; Chipara, Alin Cristian; George, Tom; Chipara, Dorina Magdalena; Ibrahim, Elamin E.; Lozano, Karen; and Sellmyer, David J., "Fourier transform infrared spectroscopy and wide-angle X-ray scattering: Investigations on polypropylene-vapor-grown carbon nanofiber composites" (2012).

David Sellmyer Publications. 240.

https://digitalcommons.unl.edu/physicssellmyer/240

This Article is brought to you for free and open access by the Research Papers in Physics and Astronomy at DigitalCommons@University of Nebraska - Lincoln. It has been accepted for inclusion in David Sellmyer Publications by an authorized administrator of DigitalCommons@University of Nebraska - Lincoln. 


\section{Authors}

Mircea Chipara, John Hamilton, Alin Cristian Chipara, Tom George, Dorina Magdalena Chipara, Elamin E. Ibrahim, Karen Lozano, and David J. Sellmyer 


\title{
Fourier transform infrared spectroscopy and wide-angle X-ray scattering: Investigations on polypropylene-vapor-grown carbon nanofiber composites
}

\author{
Mircea Chipara, ${ }^{1}$ John Hamilton, ${ }^{2}$ Alin Cristian Chipara, ${ }^{3}$ Tom George, ${ }^{4}$ \\ Dorina Magdalena Chipara, ${ }^{1}$ Elamin E. Ibrahim, ${ }^{2}$ Karen Lozano, ${ }^{3}$ David. J. Sellmyer ${ }^{4}$ \\ 1. Department of Physics and Geology, The University of Texas Pan American, Edinburg, Texas 78541 \\ 2. Department of Chemistry, The University of Texas Pan American, Edinburg, Texas 78541 \\ 3. Department of Mechanical Engineering, The University of Texas Pan American, Edinburg, Texas 78541 \\ 4. Department of Physics and Astronomy, University of Nebraska, Lincoln, Nebraska 68588
}

Corresponding author - M. Chipara, chipara@yahoo.com

\begin{abstract}
Fourier transform infrared (FTIR) spectroscopy and wide-angle X-ray scattering (WAXS) investigations of isotactic polypropylene (iPP)-vapor-grown carbon nanofiber (VGCNF) composites containing various amounts of VGCNFs ranging between 0 and 20 wt $\%$. are reported. The FTIR investigations were focused on the regularity bands of iPP. The FTIR data indicated a drop in the isotacticity index as the concentration of nanofibers was increased; this suggested a decrease in the crystallinity. WAXS measurements revealed a dominating $\alpha_{1}$ phase, with a small admixture of $\gamma$ phase or mesophase. The loading of the polymeric matrix with carbon nanofibers (CNFs) did not induce significant changes in the morphology of the polymeric matrix. A weak decrease in the size of a crystallites upon loading of CNFs was noticed. The experimental data obtained by FTIR spectroscopy supported the WAXS data. Spectroscopic data (a drop in the isotacticity index as estimated by FTIR spectroscopy and the ratio between the crystalline and total areas of WAXS lines assigned to iPP) failed to confirm the enhancement of the degree of crystallinity of polypropylene upon loading by nanofibers. However, whereas both techniques can identify with a high accuracy vibrations in ordered domains (FTIR spectroscopy) and the crystalline structure, including the lattice parameters and the size of crystallites (WAXS), difficulties in the correct assessment of the baseline and of amorphous components may result in important errors (typically $>5 \%$ ) in the estimation of the degree of crystallinity of the polymeric component.
\end{abstract}

Keywords: carbon nanotube, FTIR, nanocomposites, poly(propylene) (PP), WAXS

\section{Introduction}

Thermogravimetric analysis (TGA) studies demonstrated an increase in the thermal stability of isotactic polypropylene (iPP) through loading of vapor-grown carbon nanofibers (VGCNFs). Detailed analysis of the TGA data suggested a complex polymer-VGCNF interface, which consisted of a soft component, which extended about $10^{2} \mathrm{~nm}$ from the surface of the VGCNFs (a thickness that was on the order of magnitude of the radius of gyration), and a hard interface, which extended about $100 \mathrm{~nm}$ from the surface of the VGCNFs. ${ }^{1}$ The molecules of the macromolecular chains that were within this hard interface were subjected to very strong van der Waals interactions, which were equal to or stronger than the weakest covalent bond of iPP.2, 3 Consequently, the molecules located within the hard inter- face were trapped by the VGCNFs and could not escape from the nanofibers. Hence, during thermal degradation, all parts of polypropylene (PP) except the molecules located in the hard phase were volatilized. TGA ${ }^{1}$ investigations, electron spin resonance studies, ${ }^{4}$ and Raman investigations ${ }^{4}$ of iPP-VGCNF composites were reported elsewhere. ${ }^{1,4}$ Electron spin resonance spectroscopy confirmed ${ }^{4}$ the generation of free radicals during the processing of iPP-VGCNF composites, the existence of magnetic traces due to catalyst residues embedded within VGCNF, and the presence of conducting electrons delocalized over VGCNFs. The resonance line shape suggested a percolating transition from an insulator toward a conducting nanocomposite. The Raman spectroscopy data revealed ${ }^{4}$ that the addition of VGCNF to iPP dramatically affected the dynamics of the macromolecular chains. It was concluded ${ }^{4}$ that the loading 
of iPP with VGCNFs resulted in a rapid drop in the intensity of Raman lines assigned to the polymeric matrix, which was accompanied by a fast broadening of these lines. Such a fast vibrational dephasing of the macromolecular chain demonstrated the strong interaction between the VGCNFs and iPP chains, making difficult the study of both the polymeric matrix and filler over a wide range of VGCNF concentrations with Raman spectroscopy. ${ }^{4}$ In other words, the loading of iPP with VGCNFs in excess of $5 \mathrm{wt} \%$ almost completely washed the polymeric features of the Raman spectrum. Nevertheless, Raman spectroscopy provided information regarding the effect of the filler (in this case, VGCNFs) on the polymeric matrix and revealed ${ }^{4}$ an increase in the degree of disorder as the loading of VGCNFs was increased and complex changes of the Raman line positions and line widths. Because of the analogy between Raman and Fourier transform infrared (FTIR) methods, it was expected that FTIR spectroscopy could not provide new information regarding the effect of VGCNFs on the dynamics of iPP. Surprisingly, we found that FTIR investigations performed in the attenuated total reflectance (ATR) mode were an excellent tool in the analysis of the effect of VGCNFs on the polymeric matrix. Consequently, the first part of this contribution is focused on the analysis of the effect of VGCNFs on the dynamics and crystallinity of iPP, as revealed by FTIR measurements in the ATR mode, whereas the second part is concentrated on the morphology of the iPPVGCNF composites, as revealed by wide-angle X-ray spectroscopy.

FTIR is an excellent tool for the investigation of the so-called crystalline bands (associated with the intermolecular forces occurring between atoms and molecules within the crystalline lattice) and the regularity of helix bands (or regularity bands assigned to intramolecular vibrations within a given chain). ${ }^{5}$ For iPP, regularity bands are located below $1400 \mathrm{~cm}^{-1}$ and are connected to different values of the number $n$, which describes the minimum number of monomers linked in an isotactic sequence that defines a helical structure. The FTIR lines of iPP located at about 810, 841, 900, 940, 998, 1044, 1103, 1303 , and $1330 \mathrm{~cm}^{-1}$ were identified as originating from helical structures, increasing as the temperature is lowered below the melting temperature. ${ }^{5-8}$ The so-called regularity bands associated with the helical structures are responsible for the further development of crystallites located at 973, 998, 841, and $1220 \mathrm{~cm}^{-1}$ and have been assigned to chains of isotactic sequences containing 5, 10,12, and 14 monomer units, respectively. ${ }^{5,8}$ The connection between these bands and the temperature/ crystallinity has been recently demonstrated. ${ }^{5,8}$ It was reported $^{5}$ that as the helix length exceeded 12 monomers, the crystallization process was ignited. The lines located at 972, 1254, and $1295 \mathrm{~cm}^{-1}$ were enhanced as the temperature was raised (they were also connected to the helical structure). ${ }^{5,7,8}$ The line located at $830 \mathrm{~cm}^{-1}$ increased as the temperature was raised, being assigned to chains in a nonhelical configuration. ${ }^{7}$ For the line located at $1220 \mathrm{~cm}^{-1}$, there is debate about its temperature dependence. , $7,8^{-1}$ Other helical lines were noticed at 1167 and $1180 \mathrm{~cm}^{-1}$.

The lines located at 841,998 , and $1220 \mathrm{~cm}^{-1}$ have been associated with crystalline features, as they disappeared above the melting temperature. ${ }^{5}$ The line located at $973 \mathrm{~cm}^{-1}$ was observed both in the liquid and in the solid phase (its intensity decreased as the temperature was lowered from about $150^{\circ} \mathrm{C}$ ), and the lines located at 900 and $1100 \mathrm{~cm}^{-1}$ have been reported in both crystalline and liquid phases (but their intensity increased as the temperature was lowered from $150^{\circ} \mathrm{C}$ below the crystallization temperature).$^{5,8}$ FTIR data on PP and PPnanoclay composites revealed that the addition of nanoclays affected the absorption bands located at 973 and $995 \mathrm{~cm}^{-1}$, assigned to the deformation of tertiary methyl groups. ${ }^{9}$ A very weak line was also noticed at about $940 \mathrm{~cm}^{-1}$ and was assigned to CC chain motions. ${ }^{7}$ A relatively broad and weak line was noticed around 872 $\mathrm{cm}^{-1}$. Strong ATR lines have been observed at $806 \mathrm{~cm}^{-1}$ (assigned to CC motions), $839 \mathrm{~cm}^{-1}$ (eventually due to isomeric defects), and $897 \mathrm{~cm}^{-1}$.

Wide-angle X-ray scattering (WAXS) measurements were performed to analyze the effect of VGCNFs on the crystallinity of iPP. WAXS revealed up to four crystalline phases in iPP:10-15 a smecticlike phase and three crystalline phases identified as $\alpha, \beta$, and $\gamma$, respectively. The chain conformation of each crystalline phase is a 3-1 helix. The a phase has a monoclinic symmetry ${ }^{10-16}$ and does not require special conditions to nucleate and grow. Technically, there are two a phases with similar X-ray diffraction patterns. In the $a_{1}$ phase, the lines characterized by $h+k=$ odd (231 and 161) are absent, whereas in the $a_{2}$ phase, these lines are present. ${ }^{12-14}$ The other X-ray lines are identical to both phases. ${ }^{12-14}$ The $\beta$ phase, characterized by a (pseudo)hexagonal symmetry, ${ }^{9,10,17}$ has been noticed only under special crystallization conditions, at high undercooling, or if special nucleating agents (e.g., $\left.\mathrm{SiO}_{2}^{9}\right)$ were dispersed within the polymeric matrix. ${ }^{9}, 17$ The $\gamma$ phase was initially assigned to a triclinic symmetry; ${ }^{10}$ recent investigations revealed for this phase a face-centered orthorhombic cell with the most important lines located at (in $2 \theta$ for $\mathrm{Cu} \mathrm{Ka)} \mathrm{13.5,} \mathrm{16.3,} \mathrm{19.5,} \mathrm{20.6,} \mathrm{21.2,} \mathrm{23.6,} \mathrm{and} \mathrm{24,5}{ }^{\circ}{ }^{14}$ This phase has been obtained at high pressures. ${ }^{9,17}$ At normal pressure, the $\gamma$ phase melts at $187.2^{\circ} \mathrm{C}$, whereas the a phase melts at $186.1^{\circ} \mathrm{C}^{12}$ By increasing the pressure, the $\gamma$ phase starts to melt and crystallize at lower temperatures than the a phase. ${ }^{10,12}$ The polymorphism of $\mathrm{PP}$ is derived from different packings of the helix into the unit cell. The amorphous phase is responsible for a broad line (halo) located at about $17^{\circ} \cdot{ }^{18}$ Typically, the a phase of iPP coexists with amorphous structures. ${ }^{19}$ 
It is generally recognized that the physical properties of nanocomposites are significantly affected by the polymer-nanofiller interface. ${ }^{6-18}$ The interactions between the nanofiller and macromolecular chains affect the overall crystallinity of nanocomposites. Nevertheless, the effect of nanofillers on the crystallization process is not completely understood. Most articles ${ }^{15,} 20,21$ report a faster crystallization of polymers filled with nanoparticles and suggest that the nanoparticles may act as nucleating/crystallization centers. The effect of nanofiller on the equilibrium degree of crystallinity of polymeric samples has not been systematically investigated. Although the general perception ${ }^{21}$ is that the nanofiller increases the degree of crystallinity of the polymeric phase, the as-obtained experimental results are scattered. ${ }^{20,}{ }^{21}$ Studies on syndiotactic polypropylene (sPP) filled with carbon nanotubes showed that the thickness of the sPP crystallite along a direction perpendicular to [200] decreased with the concentration of dispersed nanotubes for samples quenched at $25^{\circ} \mathrm{C} .{ }^{11} \mathrm{~A}$ weak increase in the thickness of the sPP crystallite as the loading of nanotubes was increased was reported ${ }^{11}$ for the same nanocomposites quenched at $100^{\circ} \mathrm{C}$

In the case of iPP loaded with multiwalled carbon nanotubes (MWNTs), only the WAXS lines specific to the a phase have been reported. ${ }^{16}$ Some authors concluded that MWNTs act as nucleating agents for the crystallization of the $\alpha$ and $\gamma$ phases of iPP. ${ }^{15,}{ }^{18-25} \mathrm{Re}-$ cent studies have suggested that carbon nanotubes accelerate the crystallization of PP if their concentration is below the critical concentration for gelation. ${ }^{23}$ Above this concentration, the increase of the crystallization rate of the polymeric matrix due to carbon nanotubes is within experimental error. Investigations of the crystallization of iPP at high cooling rates revealed the formation of a dominant a phase and a mesomorphic phase responsible for two lines, one located at $15.5^{\circ}$ and the other at $21.0^{\circ}$ (in $2 \theta$ for $\mathrm{Cu} \mathrm{Ka}$ ). ${ }^{17}$ The WAXS lines of the a phase were noticed at the following angles (in $2 \theta$ for $\mathrm{Cu} \mathrm{Ka}$ ):14.1, 16.9, 18.6, 21.2, 22.1, 25.5, and $28.5^{\circ 17,18}$ and at 13.0, 16.5, 18.1, 20.7, 21.2, 21.4, 24.8 , and $27,7^{\circ} .{ }^{19}$ The weight of the mesomorphic phase increased as the cooling rate was increased. The transition from the mesomorphic phase toward the a phase started at about $45^{\circ} \mathrm{C} .^{18}$

\section{Experimental}

iPP-VGCNF nanocomposites were obtained as reported elsewhere. ${ }^{1,4}$ Composites loaded with various amounts of VGCNFs ranging between 0 to $20 \%$ were prepared. FTIR/ATR investigations were performed with a (Bruker Optics, Billerica MA, USA and Ettlingen, Germany) Equinox 55 FTIR spectrometer equipped with mercury cadmium telluride (MCT) liquid cooled

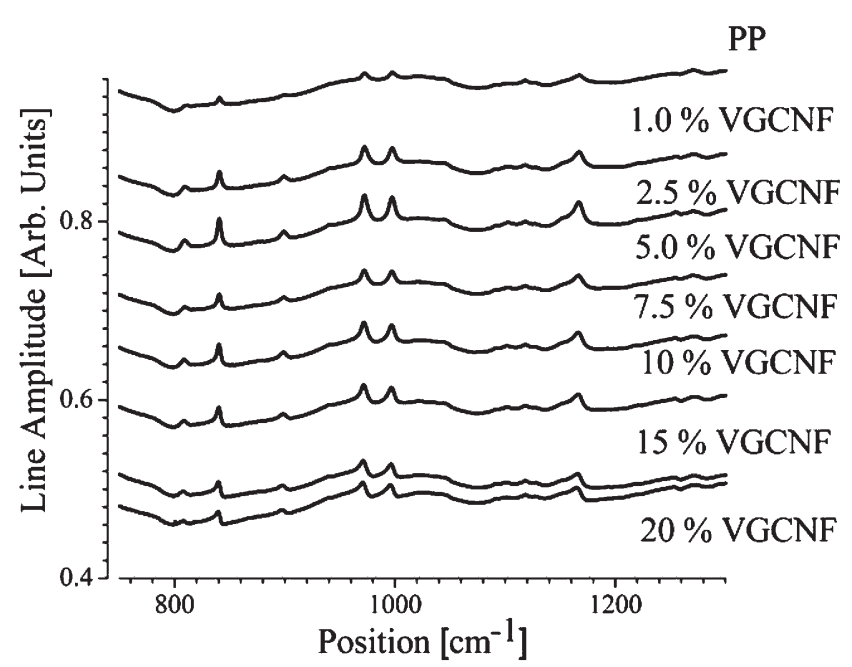

Figure 1. FTIR spectra of the iPP-VGCNF composites in the range $750-1500 \mathrm{~cm}^{-1}$.

nitrogen detector with a SpectraTech horizontal ATR instrument with a ZnSe crystal. WAXS scattering investigations were performed with a Rigaku D/Max-B diffractometer operating at a $\mathrm{Cu} \mathrm{Ka}$ wavelength $(\sim 1.544$ $\AA$ A).

\section{Results and Discussion}

\section{FTIR investigations}

As shown Figure 1, the FTIR spectra of the iPPVGCNF composites in the range $750-1500 \mathrm{~cm}^{-1}$ was rather complex, with over 10 signals identified.

However, the lines located at 871, 1021, 1058, 1116, and $1129 \mathrm{~cm}^{-1}$ were not identified. As these lines were noticed even in the pristine polymer, they may have reflected defects or impurities present within the polymeric matrix. Consequently, the most intense crystalline bands were observed in the range $800-1020 \mathrm{~cm}^{-1}$. The FTIR spectra ranging from 800 to $1020 \mathrm{~cm}^{-1}$ were simulated to quantitatively analyze the effect of the loading of VGCNFs on the FTIR lines of iPP. The simulation was carried out in Origin (OriginLab Corporation, One Roundhouse Plaza, Suite 303, Northampton, MA 01060, USA) 8 Pro with a small routine written in $C$ with the assumption that the as-recorded spectrum was a convolution of seven Lorentzian lines. The envelope of the simulated spectrum had an intensity $(I)$ defined by

$$
I(x)=\sum_{i=1}^{l} \frac{A_{i}^{\mathrm{FTIR}}}{\left[1+\left(\frac{x-x_{0 i}}{\delta_{i}}\right)^{2}\right]}+B_{0}+B_{1} x
$$

where $x$ is the actual wave number, $A_{i}^{\text {FTIR }}$ is the amplitude, $x_{o i}$ is the position, and $\delta_{i}$ is the width of each component. The subscript $i$ identifies the component lines 


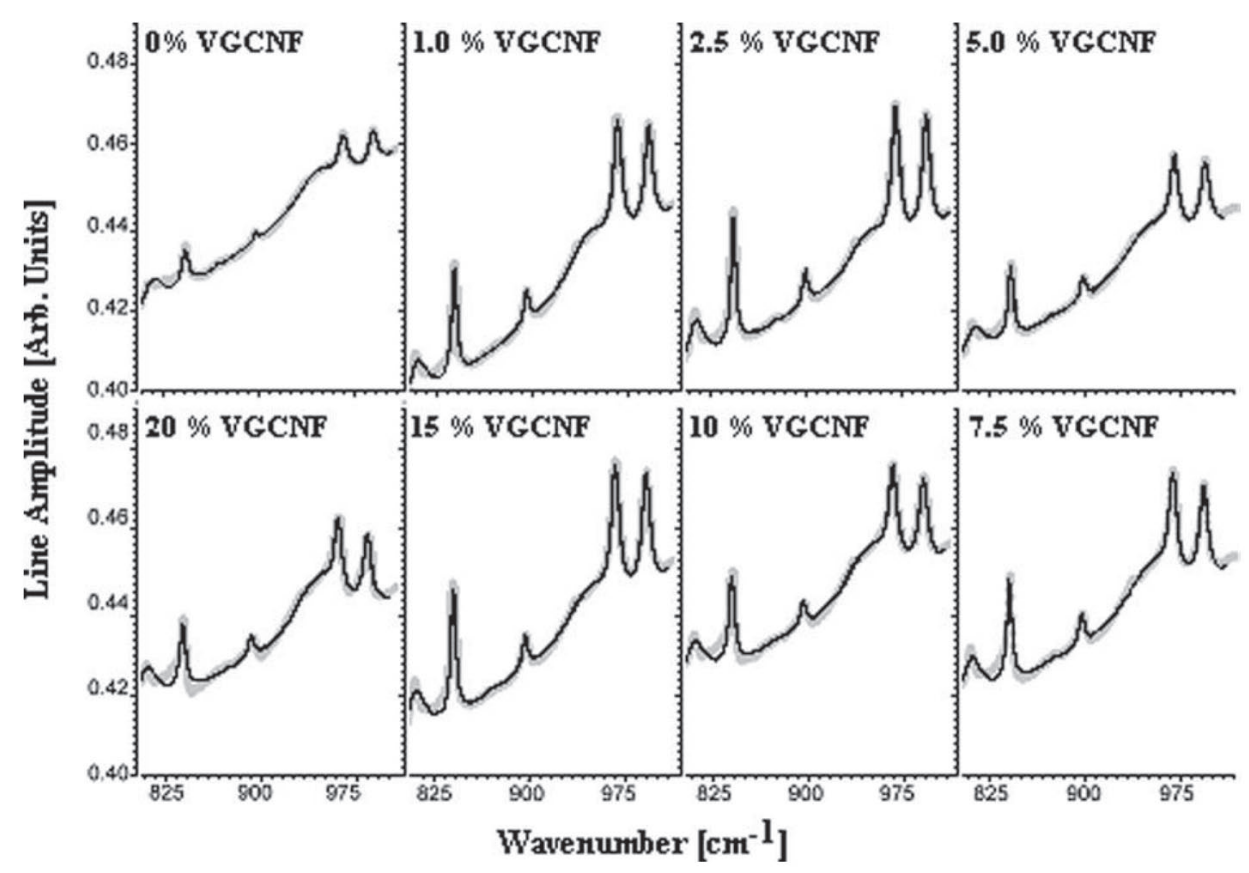

Figure 2. FTIR spectra of the iPP-VGCNF composites containing various amounts of VGCNFs. The gray points represent the experimental data, and the narrow black line represents the best fit.

(with values from 1 to 7). Baseline correction (zero position and slope) was also included. As shown in Figure 2 , Eq. (1) result in a very good simulation of the experimental data (the wide gray line represents the experimental data, and the narrow darker line represents the best fit).

The line located at $830 \mathrm{~cm}^{-1}$, reported in thin films of iPP deposited from solution, ${ }^{5-8}$ was observed in this work at $840 \pm 2 \mathrm{~cm}^{-1}$ in the thick films of iPP and iPPVGGCNFs obtained by extrusion. The shift of the position of this line toward higher wavelengths may have originated from differences in the average molecular masses of these samples, different histories (solution vs solid processing), and the highest concentration of chain entanglements in films obtain by extrusion compared to the case of thin films obtained from solution. This line is typically assigned to chains in a nonhelical configuration $^{7}$ and may be explained by the heating of the iPP-VGCNFs above $120^{\circ} \mathrm{C}$ during sample preparation and subsequent freezing by fast cooling. Nevertheless, the processing of the iPP-VGCNFs did not include cooling below the glass-transition temperature. The ratio between the areas of the lines located at 841 and at $973 \mathrm{~cm}^{-1}$ increased as the concentration of VGCNFs increased above $7.5 \%$ (see Fig. 4, shown later). This suggested a decrease in the concentration of helical structure due to the addition of nanofibers. To obtain a refined picture of the effect of VGCNFs on the isotacticity index of PP, the FTIR lines located at 973 and 998 $\mathrm{cm}^{-1}$ were isolated and simulated by an overlap of two
Lorentzian lines (including zero and slope corrections for the baseline).

As shown in Figure 3, accurate simulations of the experimental spectra were obtained. The ratio between the surface of the peak located at $998 \mathrm{~cm}^{-1}$ and the area of the peak located at $973 \mathrm{~cm}^{-1}$ (see Fig. 4) decreased as the concentration of VGCNFs was increased (the intensity of the peak located at $998 \mathrm{~cm}^{-1}$ above the melting temperature was zero ${ }^{8}$ ). This ratio defines the isotacticity in$\mathrm{dex}^{24}$ for iPP and suggested that the degree of crystallinity of PP decreased with the loading of VGCNFs.

In conclusion, the iPP-VGCNF composites contained large crystallites as well as very short crystalline domains and helical structures because of the freezing of the polymer below the melting/crystallization temperature. Structures containing helices consisting of only a few monomers were observed.

\section{WAXS investigations}

WAXS of iPP-VGCNF composites loaded with 0 to $20 \mathrm{wt} \%$ carbon nanofibers (CNFs) were recorded at room temperature and are collected in Figure 5.

The most intense lines were located between 13 and $22^{\circ}$ (in 20), and most of them were unequivocally assigned to a monoclinic a phase, with $a=0.66 \mathrm{~nm}, b=$ $0.65 \mathrm{~nm}, c=2.1 \mathrm{~nm}$, and $\mathrm{a}=99^{\circ} 20^{\prime} .{ }^{15}$ The [040] reflection was the most intense line of the spectrum. All of the investigated samples contained mostly the $\alpha_{1}$ phase (no lines were observed in the range $30-32^{\circ}$ ); this suggested a random orientation of methyl groups. ${ }^{13,14}$ This 


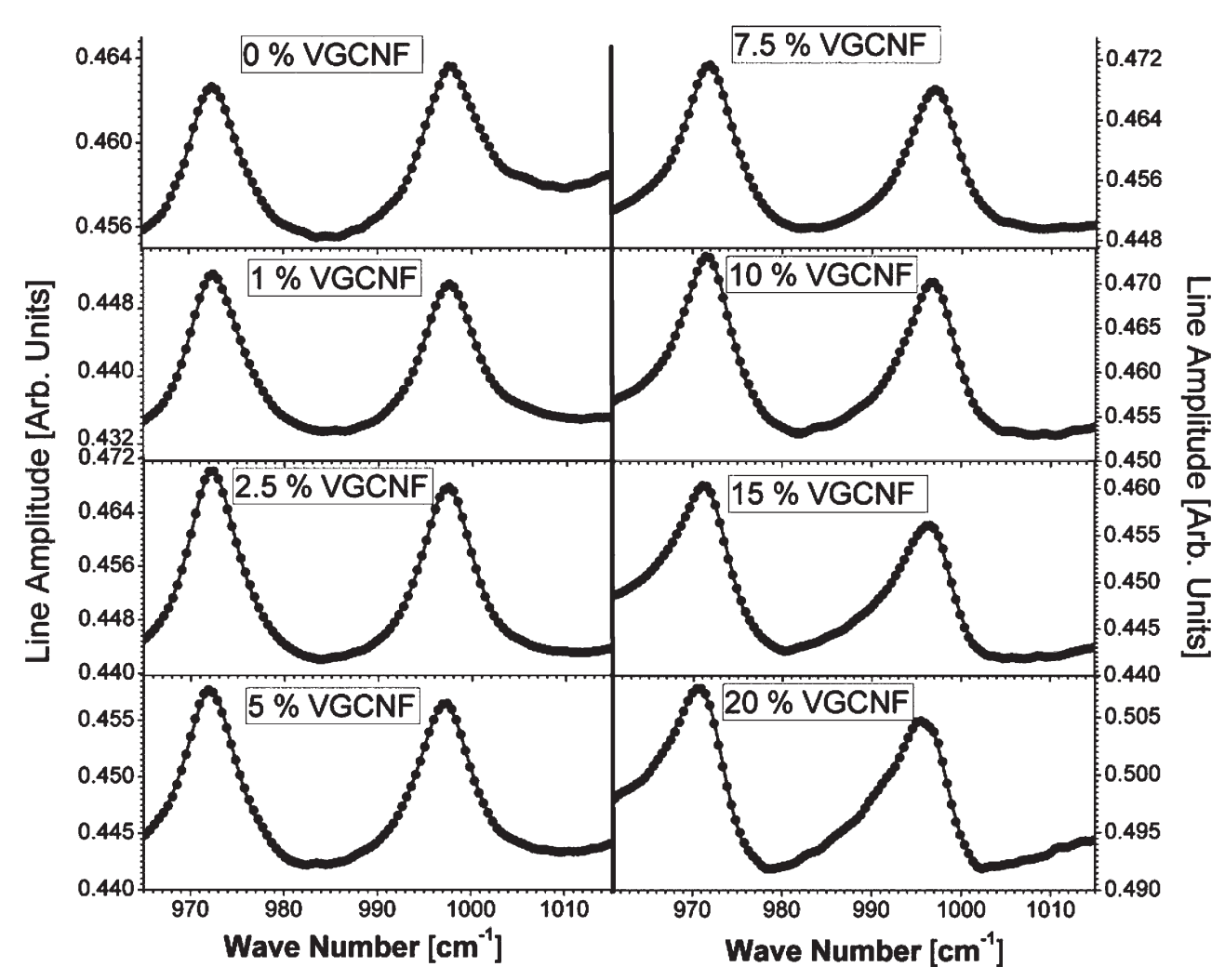

Figure 3. FTIR spectra of the iPP-VGCNF composites containing various amounts of VGCNFs. The gray points represent the experimental data, and the narrow black line represents the best fit.

was expected, as previous studies of iPP loaded with MWNTs revealed the existence of a single a phase. ${ }^{20}$ An additional weak and broad line located at $19.7 \pm 0.2^{\circ}$ (in 2 $\theta$ ) that could not be assigned to a iPP was also noticed. This line could be assigned either to the $\gamma$ phase of $\mathrm{iPP}^{9,10,22}$ or to a mesostructure ${ }^{18}$ with the character-

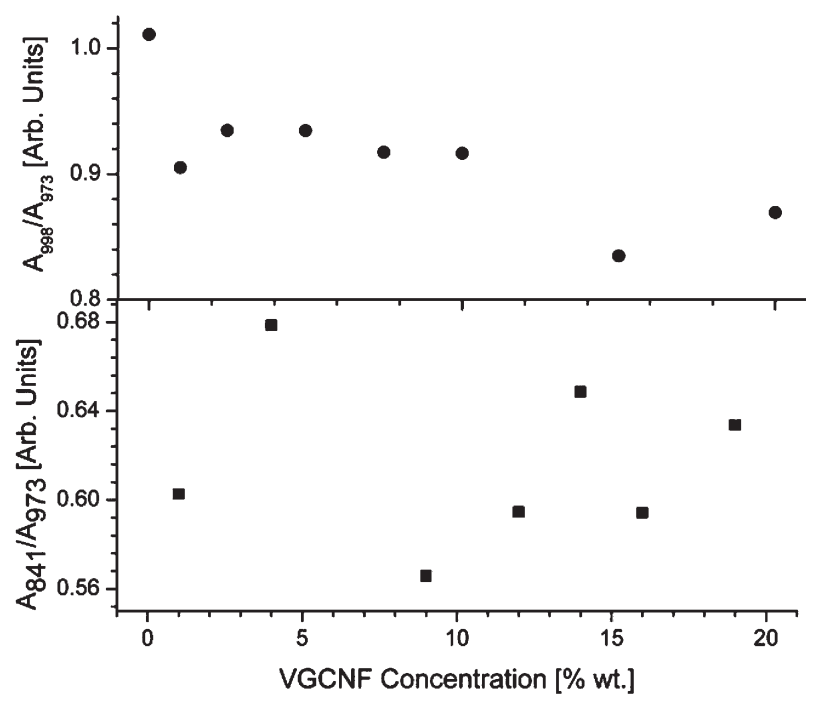

Figure 4. Dependence of the ratio between the area of the FTIR peak located at $998 \mathrm{~cm}^{-1}$ and the peak located at $973 \mathrm{~cm}^{-1}$ (top) and of the ratio between the area peak located at 841 and at $973 \mathrm{~cm}^{-1}$ (bottom) on the loading of VGCNFs. istic WAXS line located at about $20^{\circ}$. Although $\gamma$ phase appears typically in iPP at high pressures, recent studies ${ }^{9}$ revealed the presence of the $\gamma$ phase at relatively low pressures in iPP-nanoclay composites. ${ }^{9}$ However, it is important to mention that in nanocomposites, the local stresses acting on macromolecular chains could be

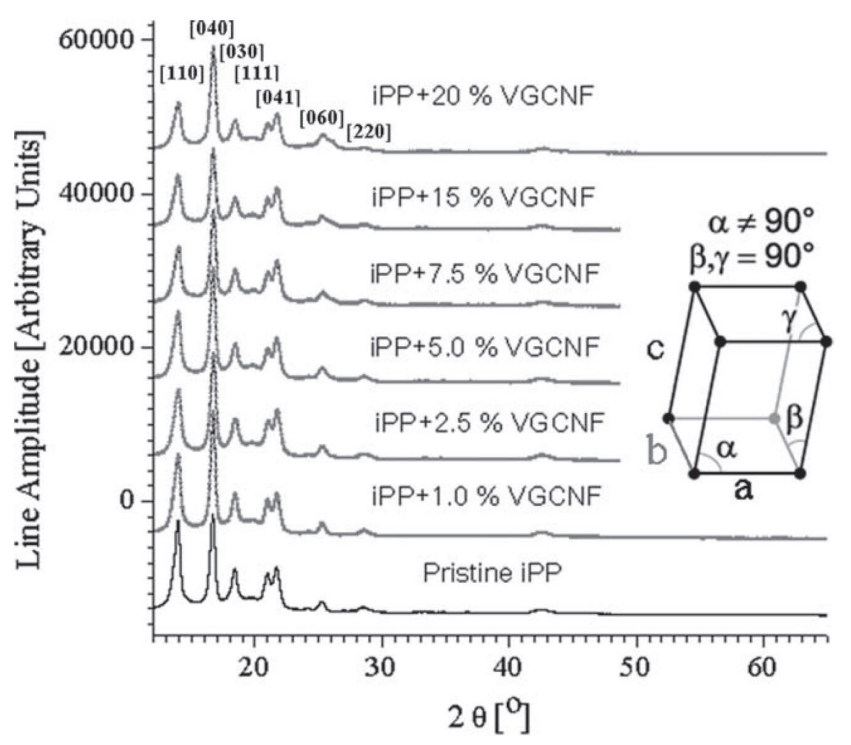

Figure 5. WAXS spectra of iPP-VGCNF composites showing the assignment of most important lines due to the a phase of iPP and the unit cell (see inset). 

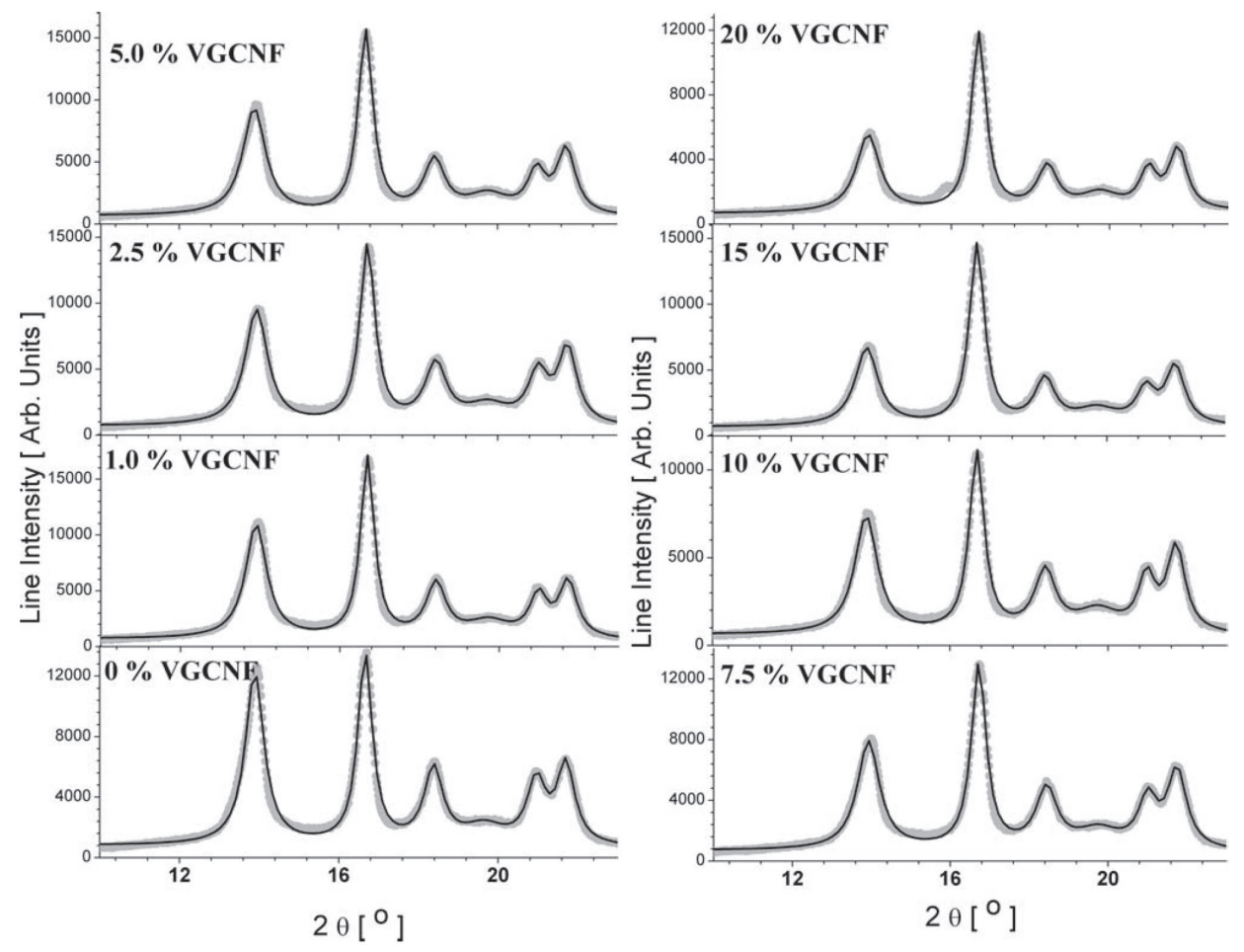

Figure 6. As-recorded (gray points) and simulated WAXS spectra (black line) of the iPP-VGCNF composites.

significantly large. Raman spectroscopy revealed important stresses acting on carbon nanotubes and nanofibers dispersed within polymeric matrices. ${ }^{2}$ The WAXS line of the CNFs (located at about $22^{\circ}$ in $2 \theta$ ) was weak and overlapped with the diffraction line of the polymeric matrix. Within experimental error, no contributions coming from the $\beta$ phase were recorded in any of the samples belonging to the iPP-VGCNF series. ${ }^{9}$

For a more detailed investigation of the effect of VGCNFs on the crystalline organization of the macromolecular chains, the WAXS spectra (ranging between 10 and $25^{\circ}$ in $2 \theta$ ) were fitted by a superposition of up to six Lorentzian lines:

$$
I(x)=\sum_{i=1}^{6} \frac{A_{i}}{\left[1+\left(\frac{x-x_{0 i}}{\delta_{i}}\right)^{2}\right]}+B_{0}+B_{1} x
$$

where $A_{i}, x_{o i}$ and $\delta_{i}$ are the amplitudes, positions, and widths of the six lines considered in this simulation. $I(x)$ is the amplitude of the as-recorded spectrum at a given $2 \theta$ angle defined as $x . B_{0}$ and $B_{1}$ are constants introduced for baseline correction (position and slope). Five of these lines were identified as belonging to the a phase of iPP.

As seen from Figure 6, the WAXS spectra of the iPPCNF nanocomposites were successfully simulated with Lorentzian lines (the thin line represents the best fit, whereas the gray dots are associated with the experimental data). This analysis resulted in an accurate estimation of the resonance line parameters. Figure 7 depicts the changes in the crystalline parameters (line positions) due to the loading of VGCNFs. Within experimental error, the positions of all of the diffraction lines were not affected by the loading of CNFs.

This suggested that the VGCNFs did not penetrate within the crystalline lattice of the polymeric matrix. With the size of the unit cell (for iPP, on the order of $10-10^{1} \mathrm{~nm}$ ) and the diameter of the VGCNFs (on the order of $10^{2} \mathrm{~nm}$ ) taken into account, it became obvious that VGCNFs did not have sufficient room to penetrate within the polymeric crystal. Hence, the polymer crystallites may have grown from the surface of the

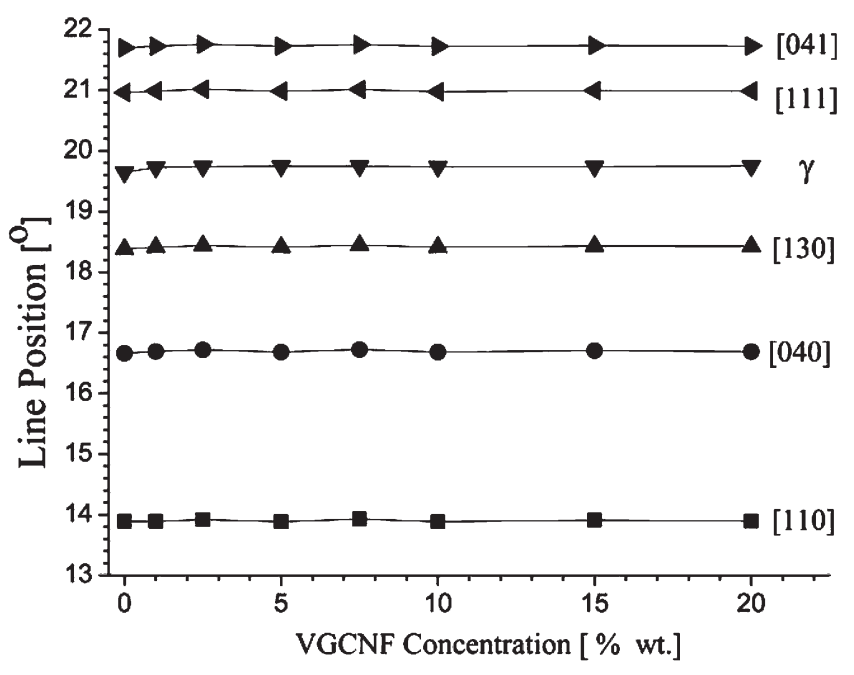

Figure 7. Dependence of the WAXS line position on the VGCNF loading. 


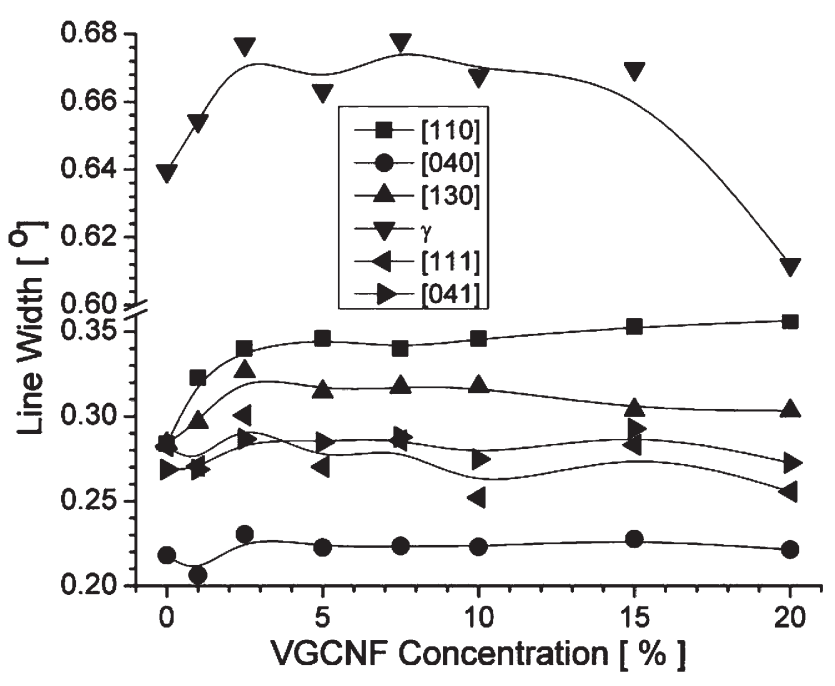

Figure 8. Dependence of the WAXS line width on the VGCNF loading.

VGCNFs but could not accommodate the VGCNFs. At a high concentration of VGCNFs, the iPP crystallites became smaller and smaller, as they started to collide more and more frequently with the filler.

The line width of the a phase showed an increase with the loading of VGCNFs, eventually toward an asymptotic value (see Fig. 8). The WAXS line width is connected to the reciprocal crystallite size along a direction perpendicular to the direction responsible for the diffraction line by the Scherrer equation:

$$
L_{h k l}=\frac{k \lambda}{\delta_{h k l} \cos \theta}
$$

where $k$ is the crystallite shape factor, $L_{h k l}$ is the size of the crystallite in a direction perpendicular to the reflection plane $(h k l), \lambda$ is the wavelength of the incoming beam $(0.1544 \mathrm{~nm}), \delta_{h k l}$ is the width of the line at halfmaximum intensity, and $\theta$ is the Bragg angle.

As shown in Figure 8, the width of the line assigned to the $\gamma$ phase showed an opposite dependence on the concentration of VGCNFs; this suggested that the size of these crystals increased as the concentration of VGCNFs was increased. Raman data ${ }^{2}$ indicated that the macromolecular chains wrapped around the VGCNFs were stretched; this suggested that the initial nucleation involved a $y$ phase that executed a fast conversion into an $\mathrm{a}_{1}$ phase during processing.

The line width assigned to the $\gamma$ phase showed a more complicated dependence on the concentration of CNFs. For a low concentration of CNFs, an increase in the CNF loading resulted in a weak increase in the line width; this suggested a weak compression of these crystallites. However, as the concentration of CNFs was increased above $1 \mathrm{wt} \%$, the lines assigned to the $\gamma$ phase started to narrow; this indicated that the crystallites be- came larger as the CNF concentration was increased. We speculated that these crystals grew at the VGCNFiPP interface during the processing step. As y phase was not thermodynamically stable, the stress relaxations and temperature converted it into the a phase. We speculated that, eventually, the $\gamma$ phase was ignited by molecules of the polymeric chains that were captured within the so-called hard interface. ${ }^{1}$

The need for a complex interface has been discussed extensively elsewhere. ${ }^{17-19}$ Previous TGA studies ${ }^{1}$ on the same samples revealed two interphases: a hard and thin interphase represented by macromolecular chains captured by CNFs at such small distances that the Van der Waals interaction of these polymeric segments with the CNFs were stronger that the covalent bonds that connected the monomers within the polymeric chain and a soft and thick elastic interface. The polymeric segments anchored in the soft phase introduced a perturbation in the morphology of macromolecular chains over a distance comparable to the radius of gyration of the polymeric matrix. The crystalline phase should have also been located within such a range to sense the contribution of CNFs. Hence, the elastic phase should have had a substructure that included an amorphous layer, which connected and matched the hard interface to the external crystallite.

\section{Conclusions}

- The FTIR data confirmed the presence of the so-called regularity bands in the iPP-VGCNF composites and showed that the isotacticity index of iPP decreased as the concentration of VGCNFs was increased. This suggested a drop in the degree of crystallinity upon the loading of the polymeric matrix by VGCNFs; this was supported by WAXS data.

- The loading of iPP by VGCNF disturbed the a crystallites, decreasing slightly and almost isotropically the size of a crystallites. Such a result suggested that the growth of a crystals was perturbed (stopped) by the addition of VGCNFs. Two simple descriptions can explain (and eventually compete with) this outcome:

1. The average size of these crystallites decreased because they started to collide with VGCNFs. Because the drop in the a-crystallites size started at low concentrations of VGCNFs, this description implies that the VGCNFs did not favor the growth of extended crystalline domains. Most of the literature agrees with this behavior.

2. The macromolecular chains and the VGCNFs were entangled. As a result, there was a pressure that VGCNFs exerted on the macromolecu- 
lar chains (the pressure exerted by the macromolecular chains on the VGCNFs was documented by Raman spectroscopy). This stress finally broke the polymeric crystallites, which (partially) relaxed the stress exerted upon the macromolecular chains. This hypothesis was consistent with the drop in the length of the crystallites as the loading of nanofillers was increased.

- The growth of a small fraction of $y$ phase was also noticed. Typically, the $\gamma$ phase requires both pressure and temperature to grow. ${ }^{17-19}$ Such conditions could appear during the processing of the polymer-filler mixture and, consequently, were not directly connected to the presence of the VGCNFs. The exceptional thermal conductivity of the VGCNFs could have also played an important role in the dissipation of heat during processing.

- The combined crystallization of the a and $\gamma$ phases was reported in PP with statistically distributed stereo errors, which for our samples, could be generated during the processing step. ${ }^{26}$

There is a tendency in the literature on polymerbased composites ${ }^{21-28}$ to consider that nanometersized fillers have a complex behavior, acting as nucleating agents, reducing the induction time, ${ }^{28}$ decreasing the crystallization temperature, lowering the enthalpy of fusion, increasing the crystallization speed, resulting in larger crystallites, and leading to a higher degree of crystallinity. Such indiscriminate and often not properly subtantiated conclusions should be discussed and analyzed with more caution. Eventually, discrepancies in the estimation of the degree of crystallinity and of the enthalpy of mixing reflect an improper calculation that did not recognize that the crystallization process occurs solely in the polymeric phase and not in the whole volume of the composite. Typically, it is assumed that MWNTs are a-nucleating agents. ${ }^{20}$ This should be also the case for VGCNFs. Literature on PP loaded with various fillers has suggested that the degree of crystallinity is not affected by $\mathrm{MWNTs}^{20}$ or revealed an increase in the degree of crystallinity upon loading of carbon nanoparticles. ${ }^{21}$ However, FTIR data originating solely from the polymeric component suggested a decrease in the isotacticity index by about $10 \%$ upon the loading of iPP with 20 wt \% VGCNFs. Further studies on the crystallization of PP-VGCNFs by DSC techniques, which will complement existing data, ${ }^{27}$ are being done.

\section{References}

1. Chipara, M. D.; Lozano, K.; Hernandez, A.; Chipara, M. Polym Degrad Stab 2008, 93, 871.

2. Ajayan, P. M.; Schadler, L. S.; Giannaris, C.; Rubio, A. Adv Mater 2000, 12, 750.

3. Jiang, L. Y.; Huang, Y.; Jiang, H.; Ravichandranc, G.; Gao, H.; Hwang, K. C.; Liu, B. J Mech Phys Solids 2006, 54, 2436.

4. Chipara, M.; Villarreal, J. R.; Chipara, M. D.; Lozano, K.; Chipara, A. C.; Sellmyer, D. J. J Polym Sci Part B: Polym Phys 2009, 47, 1644.

5. Zhu, X.; Yan, D.; Fang, Y. J Phys Chem B 2001, 105, 12461.

6. Singh, C.; Kaur, S.; Kothari, A. V.; Naik, D. G.; Vyas, P. B.; Gupta, V. K. J Appl Polym Sci 2009, 113, 3181.

7. Khafagy, R. M.;Badr. Y. A. J Polym Sci Part B: Polym Phys 2005, 43, 2829.

8. Brookes, A.; Dyke, J. M.; Hendra, P. J.; Meehan, S. Spectrochim Acta A 1997, 53, 2313.

9. Yuan, Q.; Rajan, V. G.; Misra, R. D. K. Mater Sci Eng B 2008, 153,88 .

10. Bond, E. B.; Spruiell, J. E.; Lin, J. S. J Polym Sci Part B: Polym Phys 1999, 37, 3050.

11. Gorrasi, G.; Romeo, V.; Sannino, D.; Sarno, M.; Ciambelli, P.; Vittoria, V.; De Vivo, B.; Tucci, V. Nanotechnology 2007, 18, 275703.

12. Mezghani, K.; Phillips, P. J. Polymer 1998, 39, 3735.

13. Radhakrishnan, J.; Ichikawa, K.; Yamada, K.; Toda, A.; Hikosaka, M. Polymer 1998, 39, 2995.

14. Naiki, M.; Kikkawa, T.; Endo, Y.; Nozaki, K.; Yamamoto, T.; Hara, T. Polymer 2000, 42, 5471.

15. Assouline, E.; Grigull, S.; Marom, G.; Wachtel, E.; Wagner, H. D. J Polym Sci Part B: Polym Phys 2001, 39, 2016.

16. Seo, M.-K.; Lee, J.-R.; Park, S.-J. Mater Sci Eng A 2005, 404, 79.

17. Coccorullo, I.; Pantani, R.; Titomanlio, G. Polymer 2003, 44, 307.

18. Konishi, T.; Nishida, K.; Kanaya, T. Macromolecules 2006, $39,8035$.

19. Boger, A.; Heise, B.; Troll, C.; Marti, O.; Rieger, B. Eur Polym J 2007, 43, 634.

20. Ganß, M.; Satapathy, B. K.; Thunga, M.; Weidisch, R.; Potschke, P.; Jehnichen, D. Acta Mater 2008, 56, 2247.

21. Vassiliou, A.; Bikiaris, D.; Chrissafis, K.; Paraskevopoulos, K. M.; Stavrev, S. Y.; Docoslis, A. Compos Sci Technol 2008, $68,933$.

22. Zhang, S.; Minus, M. L.; Zhu, L.; Wong, C-P.; Kumar, S. Polymer 2008, 49, 1356.

23. Xu, D.; Wang, Z. Polymer 2008, 49, 330.

24. Russell, C. A. J Appl Polym Sci 1960, 4, 219.

25. Wang, Z. G.; Hsiao, B. S.; Sirota, E. B.; Agarwal, P.; Srinivas, S. Macromolecules 2000, 33, 978.

26. Hild, S.; Boger, A.; Troll, C.; Rieger, B. Polym J 2009, 41, 993.

27. Raimo, M. Polym J 2011, 43, 78.

28. Chen, X.; Wei, S.; Yadav, A.; Patil, R.; Jiahua Zhu, J.; Ximenes, R.; Sun, L.; Guo, Z. Macromol Mater Eng 2011, 296, 434. 